\title{
An Energy Efficient Clustering Algorithm based on Boundary Nodes Allocation for WSN
}

\author{
Tanuja Dogra \\ M.E. Student \\ ECE Department \\ National Institute of Technical Teachers Training and Research (NITTTR) \\ Chandigarh, India
}

\begin{abstract}
Instead of various advantages of wireless sensor networks, there is always a constraint related to energy consumption resulting in reduced lifetime of a wireless sensor network. In this paper, an energy efficient algorithm is proposed considering allocation of boundary nodes, removing clashes among clusters while distributing nodes thereby reducing overall energy consumption in the network as compared to LEACH (Low energy adaptive clustering hierarchy)and EELEACH-MIMO (Energy Efficient LEACH multiple-inputmulti-output) schemes. The selection of the cluster head is done on the basis of residual energy of nodes in a cluster as done in LEACH. The simulation results shows that the proposed algorithm is more energy efficient and works for longer time period.
\end{abstract}

\section{General Terms}

Sensor Nodes, Clustering, Wireless Sensor Networks.

\section{Keywords}

Boundary sensor nodes, cluster heads, LEACH, Network Lifetime.

\section{INTRODUCTION}

A wireless sensor network is an outstanding technology which is growing day by day to meet the present era demands even in harsh environments. The wireless sensor networks can work more efficiently in harsh areas but the network lifetime is very much limited. So, routing in an efficient manner is the best alternative in order to increase the network lifetime considerably. Hence, it is necessary to design energy efficient routing protocols for wireless sensor networks [1-3].

Clustering is one of the various effective techniques used in wireless sensor networks to reduce energy consumption of the overall network. One of the well-known cluster based routing protocol is LEACH in which cluster formation is followed by cluster head selection. But LEACH suffers from various disadvantages which are improved to a great extent in EELEACH, LEACH-MIMO, EE-LEACH-MIMO, etc. in terms of energy efficiency and network lifetime [4]. The EELEACH protocol considers the residual energy and location of sensor nodes while selecting cluster heads. In the base paper, author has combined the EE-LEACH protocol with MIMO technology in order to enhance the network lifetime. In this scheme, there is direct communication between member nodes and cluster heads and then cluster head transmits the data in single hop consuming comparatively more energy [59].

In this paper, an energy efficient algorithm is proposed in which sensor nodes present at the boundary of two clusters are placed in a single cluster removing them from the another in such a way that the overall energy of the cluster and the network get reduced and the network remains balanced. The boundary nodes are put in a particular cluster depending upon the weighted sum metric of the clashing clusters. Finally, the clash among clusters is reduced and redundancy due to nodes lying on the boundary in the network is also removed. The overall description of the algorithm is described in following sections.

\section{PROPOSED ALGORITHM}

In this section an energy efficient routing algorithm is proposed. In the beginning $\mathrm{N}$ nodes are deployed randomly in different sectors using expression $2 \pi / \mathrm{K}$ opt. The sink is placed outside the monitoring area. The various steps included in this algorithm are as follows:

\subsection{Partitioning of Monitoring Area into Sectors: Cluster Formation}

The whole monitoring area is partitioned in optimal number of sectors known as clusters given by the expression discussed above. After sectoring nodes are deployed randomly in the whole area. Dividing area into optimal clusters can save energy as well as enhance network lifetime also.

\subsection{Prediction of nodes falling into two clusters}

The next step of the algorithm is to find out the nodes which lie on the boundary of two clusters and can be treated as part of both the clusters thereby introducing redundancy in the node distribution. This situation is shown in Fig.1.

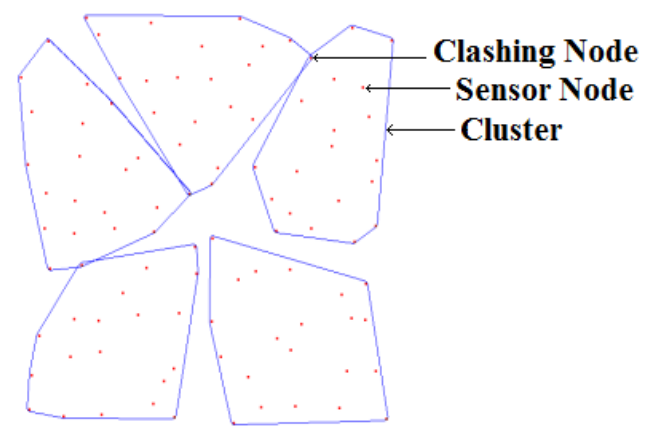

Fig.1 Clash among Clusters for the Boundary Nodes

\subsection{Removal of clash for nodes among clusters}

After predicting the nodes falling in two clusters, the next task is to place those nodes in a cluster in which overall energy consumption is less as compared to the another one which is clashing. Applying weighted sum metric, finally the clashing node is placed in that cluster for which its weighted sum is found higher. Also, the same redundant node present in the 
other cluster is removed simultaneously. This can be seen in Fig.2

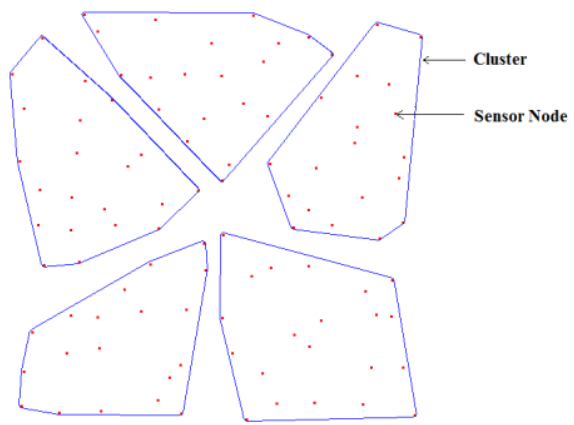

Fig. 2 Removal of clashes among clusters

\subsection{Cluster Head Selection}

In this step, a node with more residual energy is chosen as cluster head. Initially, all the nodes have almost same energies. As the number of rounds increase consecutively, the residual energy of nodes start decreasing considerably. Also, a threshold level is decided so as to choose cluster head meeting the necessary energy requirements. The packets from various nodes are collected by cluster head using basic distance formula and finally the aggregated packets are forwarded to the sink. The threshold level $b_{-t h}$ decided for a node to become cluster head is given as

b_th $=$ packet_size $*($ Etx_elec $+($ Eamp $*$ field_area $)$...

\section{CALCULATION OF ENERGY CONSUMPTION}

The equations for calculating energy required for transmission and reception are same as taken in base paper [5]. The energy consumed for transmitting a one-bit message by the transmitter at a distance $\mathrm{d}$ is given as $\mathrm{Tx} \_\mathrm{e}$,

Tx_e $=$ packet_size $*\left(E t x \_e l e x+(\right.$ Eamp $*$ d) $)$

Similarly, the receiving same message packet is given as Rx_e,

Rx_e $=$ packet size $*($ Erx_elec $)$

Where Etx_elec is transmitter circuit energy consumption per bit and Erx_elec is receiver circuit energy consumption per bit. Eamp refers to the effect of amplifier.

\section{SIMULATION AND ANALYSIS}

The analysis of energy efficiency is done in reference to EELEACH-MIMO. To evaluate the network lifetime, we consider the number of nodes still alive with the number of rounds. The value of EMt is taken as same for different Mt. The Kopt is taken as 5 in our system parameters. The various simulation parameters are given in Table. 1

Table 1. Simulation Parameters

\begin{tabular}{|c|c|}
\hline Parameter & Value \\
\hline Area of Network & $100 \times 100$ \\
\hline Number of Nodes & 100 \\
\hline Packet Size & $2000 \mathrm{bit}$ \\
\hline Position of sink & $(50,175)$ \\
\hline
\end{tabular}

\begin{tabular}{|c|c|}
\hline Initial Energy & $0.5 \mathrm{~J}$ \\
\hline $\mathrm{E}_{\mathrm{tx} \_ \text {elec }}$ & $50 \mathrm{~nJ} / \mathrm{bit}$ \\
\hline $\mathrm{E}_{\mathrm{rx} \_ \text {elec }}$ & $50 \mathrm{~nJ} / \mathrm{bit}$ \\
\hline $\mathrm{E}_{\mathrm{amp}}$ & $100 \mathrm{pJ} / \mathrm{bit} / \mathrm{m} 2$ \\
\hline $\mathrm{M}_{\mathrm{t}}$ & 2 \\
\hline $\mathrm{K}_{\mathrm{opt}}$ & 5 \\
\hline Frequency & $2.5 \mathrm{GHz}$ \\
\hline $\mathrm{d}_{\text {max }}$ & $10 \mathrm{~m}$ \\
\hline
\end{tabular}

The Table. 2 and Fig. 3 shows simulation results. The Table. 2 shows First Node Dies (FND), Half Nodes Die (HND) and Last Node Dies (LND) for LEACH, EE-LEACH, LEACH MIMO and EE-LEACH-MIMO along with proposed scheme respectively. Comparing the proposed scheme with the existing ones, it is evident that the network works considerably for a longer time and as all the nodes remain alive for a greater period, so the network is well balanced.

Table 2. FND, HND and LND for each scheme

\begin{tabular}{|c|c|c|c|}
\hline & FND & HND & LND \\
\hline LEACH* $^{*}$ & 571 & 889 & 1094 \\
\hline EE-LEACH* $^{*}$ & 932 & 1119 & 1374 \\
\hline LEACH-MIMO* $^{*}$ & 758 & 1080 & 1276 \\
\hline $\begin{array}{c}\text { EE-LEACH- } \\
\text { MIMO* }\end{array}$ & 1040 & 1274 & 1342 \\
\hline PROPOSED & 2473 & 2486 & 2506 \\
\hline
\end{tabular}

(* marked readings are taken as reference from base paper for comparing the proposed results)

The Fig. 3 shows the possible outcomes of all the existing schemes along with the proposed scheme. The network lifetime is much higher and hence is more efficient in comparison to others. This is due to even distribution of nodes as well as cluster heads which results in a well-balanced network. The curve of the proposed algorithm decays with clear steps, and is steep to a great extent which means that the consumption of energy among nodes in the network is quite balanced, thereby resulting in increased lifetime of network.

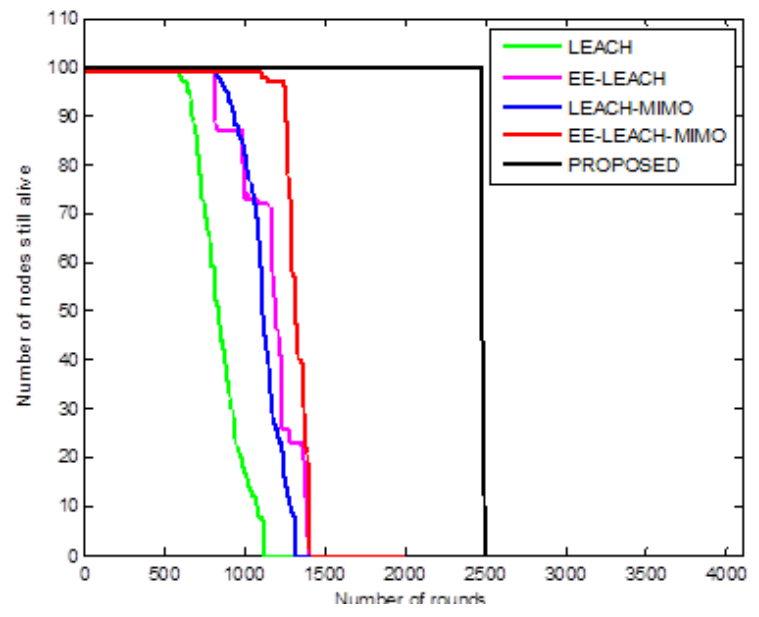

Fig. 3 The profile of network lifetime for each scheme 


\section{CONCLUSION}

The proposed scheme is simulated in MATLAB. In this paper, the redundancy introduced by the nodes lying on the boundary of two clusters is removed and hence the overall energy consumption of the network is reduced to a great extent. The network lifetime profile clearly shows that the proposed scheme has increased the lifetime of the wireless sensor network considerably in comparison to the previous existing schemes.

\section{ACKNOWLEDGMENTS}

I would like to express my sincere gratitude to my guide Prof. O.S.Khanna for the continuous support of my research work, for his motivation, enthusiasm, and immense knowledge. Besides my guide, my sincere thanks also goes to Dr.M.P. Poonia, Director NITTTR Chandigarh for providing such an inspiring work environment.

\section{REFERENCES}

[1] Kazem Sohraby, Daniel Minoli, Taieb, Znati "Wireless Sensor Networks", John Wiley \& Sons Inc. publication,Canada, ISBN No.- 978-0-471-74300-2, 2007.

[2] Ji Peng, Wu Chengdong, Zhang Yunzhou, Chen Fei, "A Low-Energy Adaptive Clustering Routing Protocol of Wireless Sensor Networks," 7th International Conference on Wireless Communications, Networking and Mobile Computing (WiCOM), pp.1-4, 23-25 Sept. 2011.

[3] H. Gou and Y. Yoo, "An Energy Balancing LEACH Algorithm for Wireless Sensor Networks" Proc. International Conference. Information Technology, pp.822-827, 2010 .
[4] B.V. Manikyala Rao, S.Pallam Setty, G.Lavanya Devi, "Energy Efficient Routing Protocols In Wireless Sensor Networks," International Journal Of Computers \& Communications, Vol. II, pp.16-23, 2013.

[5] Yongming Qin, Qiuling Tang, Ye Liang, Xiuyu Yue, Xian Li, "An Energy-Efficient Cooperative MIMO Scheme for Wireless Sensor Networks Based on clustering," IEEE 14th International Conference onComputational Science and Engineering (CSE), pp.471-474, 24-26 Aug. 2011.

[6] Jia Xu, Ning Jin, Xizhong Lou, Ting Peng, Qian Zhou,Yanmin Chen, "Improvement of LEACH protocol for WSN," 9th International Conference on Fuzzy Systems and Knowledge Discovery (FSKD),pp.21742177, 29-31May 2012.

[7] Liangrui Tang, Sheng Liu, "Improvement on LEACH Routing Algorithm for Wireless Sensor Networks,"International Conference on Internet Computing \& Information Services (ICICIS), pp.199202, 17-18 Sept.2011.

[8] Keerti Naregal, Anand Gudnavar, "Improved Cluster Routing Protocol for Wireless Sensor Network through Simplification," 18th Annual International Conference on Advance Computing and Communications (ADCOM), pp.1-3, 2012.

[9] Anjali Bharti, Kanika Sharma, "Enhancing the lifetime of LEACH Protocol using NNA for Wireless Sensor Network," International Journal of Computer Trends and Technology (IJCTT), Vol.4, pp.756-759, April 2013. 